\title{
86373 - DESNUTRIÇÃO EM NONAGENÁRIOS E CENTENÁRIOS NA COMUNIDADE: É UM PROBLEMA MULTIPROFISSIONAL?
}

\author{
Pôster - Gerontologia
}

\author{
Claudine Lamanna Schirmer / Schirmer, CL / PUCRS; Liziane da Rosa Camargo / CAMARGO, LR / PUCRS; \\ Claudia Aline Oliveira Safian / SAFIAN, CAO / PUCRS; Vanessa Binotto / BINOTTO, V / PUCRS; \\ Marlon Cassio Pereira Grigol / GRIGOL, MCP / PUCRS; Ângelo José Gonçalves Bós / BÓS, AJG / PUCRS
}

Introdução: Os nonagenários e centenários são as faixas etárias que mais crescem no Brasil, com um crescimento de 79,6\% entre os dois últimos censos. O estado nutricional é importante para qualidade de vida de idosos, mas pouco avaliada em nonagenários e centenários. Objetivo: $\mathrm{O}$ objetivo deste trabalho foi observar a prevalência de desnutrição em nonagenários e centenários e sua relação com características clínico-funcionais. Métodos: Trata-se de um estudo transversal, analítico e quantitativo. A população do estudo foi constituída por homens e mulheres com 90 anos ou mais. Resultados: Foi avaliado domiciliarmente o estado nutricional de 128 nonagenários e centenários residentes em Porto Alegre, RS, onde 31 (24\%) apresentavam-se com risco nutricional. Solteiros e viúvos apresentaram risco nutricional significativamente maior que casados. Os participantes com risco nutricional apresentaram significativamente mais sintomas depressivos, menor frequência semanal de sair de casa, menor força de preensão palmar, maior tempo para levantar-se de uma cadeira, caminhar 3 metros e retornar, referiram maior dificuldade de subir dez degraus, carregar objetos de 5 quilos, transferir-se para uma cama ou cadeira, tomar banho, vestir-se, alimentar-se e usar o banheiro sozinho $(\mathrm{p}<0,05)$. Conclusões: Concluímos que a identificação da desnutrição é um parâmetro importante para a avaliação do estado de saúde de nonagenários e centenários. Essa população demonstrou ter necessidades de cuidado específicas, entre elas a desnutrição, talvez pouco avaliada na clínica médica.

Palavras-chave: Desnutrição; Nonagenários; Centenários. 